\title{
Technical note: Use of laser capture microdissection for the localization of tissue-specific global gene expression in rumen papillae
}

\author{
M. A. Steele, ${ }^{*}$ O. AlZahal, ${ }^{*}$ S. L. Greenwood, † J. C. Matthews, $\ddagger$ and B. W. McBride ${ }^{* 1}$ \\ *Department of Animal and Poultry Science, University of Guelph, Guelph, Ontario, Canada N1G 2W1 \\ †Department of Animal Science, University of Vermont, Burlington 05405 \\ ‡Department of Animal and Food Sciences, University of Kentucky, Lexington 40546
}

\section{ABSTRACT}

Gene expression profiling of bovine rumen tissue has provided insight into dietary regulation of rumen epithelial function. However, most studies have relied on a heterogeneous sample with multiple tissue and cell types. The objective of this study was to use laser capture microdissection to characterize RNA expression profiling of epithelial and connective tissues of rumen papillae. Papillae were biopsied from 3 lactating dairy cows, frozen in cryomolds, cut into sections, stained, and dehydrated, and epithelial and connective cells were collected using laser capture microdissection. Total RNA was isolated from epithelial and connective tissue and global gene expression was assessed using the Affymetrix GeneChip Bovine Gene 1.0 ST array (Affymetrix, Santa Clara, CA). Data preprocessing was conducted using the robust multi-array average method, and detection of differentially expressed genes (DEG) was determined using ANOVA. The model included the fixed effect of tissue, and a Benjamini-Hochberg false discovery rate of 0.1 was applied to DEG. We found 382 DEG between epithelial and connective tissues. Analysis of these DEG using Ingenuity Pathway Analysis (Redwood City, CA) found that epithelial and connective tissues in rumen papillae expressed distinct RNA profiles (signatures). The epithelial signature was enriched with RNA encoding tight junction and metabolic genes, whereas connective signatures were enriched with RNA encoding proteins involved in cell structure and extracellular matrix composition. The molecular functions enriched within the top networks between the 2 tissues from the Ingenuity Pathway Analysis included connective tissue disorders, dermatological diseases and conditions, gastrointestinal disease, tissue morphology, and tissue development. In summary, it is possible to use laser capture microdissection for the localization of tissue-specific global gene expression in rumen papillae. This approach may be useful to improve the accuracy

Received April 12, 2013

Accepted September 6, 2013.

${ }^{1}$ Corresponding author: bmcbride@uoguelph.ca and interpretation of molecular measurements in future studies.

Key words: rumen papillae, epithelium, connective, gene expression

\section{Technical Note}

The ruminant forestomach is primarily known for its role in microbial fermentation, although it plays a major role in nutrient transport, metabolism, and host defense (Gäbel et al., 2002). The stratified squamous epithelium of the rumen is a heterogeneous epithelium comprising 4 distinct strata with multiple functions. The stratum corneum is the outermost layer consisting of dead cornified keratinocytes and is in direct contact with the rumen milieu and microbiota. The main role of the stratum corneum is to act as a physical protective barrier between the rumen milieu and the lower viable strata. Adjacent to the stratum corneum is the stratum granulosum, which acts as the permeability barrier of the epithelium and is characterized by its richness in junctional complexes. Beneath the granulosum are the stratum spinosum and stratum basale, which contain mitochondria, thereby contributing to the metabolic functions of the rumen epithelium. A basal lamina separates the basale cells of the mucosa from the submucosa, which comprises a heterogeneous mixture of connective tissue, collagen fibrils, and vasculature (Baldwin, 1998). The surface area of the stratified squamous epithelium is expanded by papillae, the abundance and morphology of which differs widely within and between forestomach organs.

Great progress has been made in our understanding of the structural and functional adaptations of the rumen epithelium by using histological techniques (Steele et al., 2011a). Moreover, ex vivo and in vitro techniques such as Ussing chambers (Gäbel et al., 2002) and cell culture (Baldwin, 1998) have improved our understanding of rumen function. With the rapid advancement of systems biology, many animal scientists have adopted the use of high-throughput molecular techniques to understand how bovine tissues respond to stimuli 
(Loor et al., 2013). Recent transcriptomic studies have generated hypotheses about the adaptation of the rumen epithelium to rapidly fermentable carbohydrates (Penner et al., 2011; Steele et al., 2011a,b) and during rumen development (Connor et al., 2013). In particular, transcriptomic profiling has provided insight into key processes of metabolism, nutrient transport, cellular proliferation signaling, and tight junction assembly (Penner et al., 2011).

Despite the high degree of sophistication of highthroughput functional genomic techniques, the accuracy of data interpretation is largely influenced by the tissue collection technique. With respect to the rumen epithelium, there are discrepancies in the literature regarding tissue collection methodology and dissection protocols, which limit the interpretation of the results between studies. Furthermore, the thickness of the rumen epithelium has been shown to change due to dietary treatments (Steele et al., 2011a). Thus, it may be possible that the overall difference in gene expression results, and their interpretation, reflect differences in the proportion of specific cell types that comprise a sample. Using tissue samples that are heterogeneous mixtures of cells for functional genomic studies may result in an "averaging out" of the heterogeneous elements in a given sample. For example, the lack of large RNA expression fold-changes that did not match large shifts in functional capacity found by rumen tissue transcriptomic expression profiling studies (Penner et al., 2011; Steele et al., 2011a,b) may have resulted from disproportional extraction of RNA from tissue types not responsible for the altered function.

Developing laboratory techniques to overcome the challenge of tissue heterogeneity is an essential step to further understand epithelial function when using highthroughput molecular-based techniques. Laser capture microdissection (LCM) is a technique that permits the isolation of a pure population of cells from tissue sections under microscopic visualization. Specifically, once a tissue section slide is under the LCM, a transparent ethylene vinyl acetate layer on a cap is apposed to the section and a low-power infrared laser beam is directed by the operator to isolate the cells of interest. The targeted area transiently binds the tissue and ethylene vinyl acetate, and the cells become embedded in the film for removal from the section (Curran et al., 2000). The target cells can be studied using a variety of functional genomics techniques such as transcriptomics, proteomics, and metabolomics. To date, no studies have employed LCM to dissect cell types in ruminant digestive tissues despite its potential to improve the predictability of high throughput functional genomic techniques. Taking this into consideration, the objective of this study was to develop an experimental regi- men using LCM to differentially extract epithelial and connective tissue RNA from biopsied rumen papillae of lactating dairy cattle, using resulting genomic expression profiles as indicators of the regimen's success.

All animal procedures were approved by the University of Guelph Animal Care Committee in accordance with the Canadian Council on Animal Care (1993) guidelines. Three mature, rumen-cannulated, and lactating Holstein dairy cows were maintained at the University of Guelph dairy barn. Cows were offered ad libitum access to water and a control diet of a TMR as previously described by Dionissopoulos et al. (2013).

The remainder of the protocol was conducted using RNase-free techniques to minimize RNA degradation. Rumen papillae biopsies were collected from the ventral sac using methodologies that have been previously described (Steele et al., 2011a,b). In brief, the rumen contents were partially removed to facilitate the retraction of the ventral sac to the cannula. To ensure precision of sampling location, the ventral sac was completely emptied to visualize the sampling site and only one person for all cows located and retracted the base of the ventral sac. Rumen papillae were excised using sterile surgical scissors and immediately placed in ice-cold PBS. Rumen papillae were washed 10 times in ice-cold PBS. After washing, rumen papillae were blotted dry and positioned in chilled cryomolds containing Tissue Tek OCT cryostat embedding compound (Ames Co., Division of Miles Laboratory, Elkhart, ID) placed on top of crushed ice to aid in positioning of the rumen papillae. Additional OCT cryostat embedding compound was added and the cryomold was frozen on a raft floating on top of liquid nitrogen. Frozen samples were then transported on dry ice and stored at $-80^{\circ} \mathrm{C}$ until analysis.

Within a day of collection, $10-\mu \mathrm{m}$-thick sections were cut from each cryomold using a cryostat programmed at $-20^{\circ} \mathrm{C}$ and were fixed onto prechilled microscope slides. Slides were kept either in a $-80^{\circ} \mathrm{C}$ freezer or on dry ice until their preparation for LCM. To prepare for LCM, slides were allowed to thaw just until frost disappeared, and approximately $150 \mu \mathrm{L}$ of $75 \%$ ethanol was pipetted onto sections fixed on each slide. The slides were incubated for $3 \mathrm{~min}$ at room temperature. Slides then underwent a series of washes as follows: RNaseand DNase-free water for $30 \mathrm{~s}$, hematoxylin (1:45 stain) for $30 \mathrm{~s}$, RNase- and DNase-free water for $10 \mathrm{~s}, 75 \%$ ethanol for $30 \mathrm{~s}, 95 \%$ ethanol for $30 \mathrm{~s}, 100 \%$ ethanol for $2 \mathrm{~min}$, and $100 \%$ xylene for $5 \mathrm{~min}$. The edge of each slide was tapped on a Kim-wipe tissue (KimberlyClark Professional, Roswell, GA) between each wash to remove excess liquid. The slides were then air-dried in the fumehood for $5 \mathrm{~min}$, and LCM was performed within $1 \mathrm{~h}$ of slide processing. A Veritas LCM (Mo- 
lecular Devices, Sunnyvale, CA) was used to perform LCM. The epithelial and connective tissue cells were identified based on staining (Figure 1), and the CapSure LCM transfer film was used to facilitate the isolation of cell types. The rumen epithelial and connective tissue samples (approximately 500 cells each) were dissected from a minimum of 3 papillae using a UV laser to cut the specific sections and an infrared laser to polymerize the specific tissue type to the macrocaps (Molecular Devices). Tissue captured onto the transfer film of the caps by LCM was immediately immersed in $50 \mu \mathrm{L}$ of isolation buffer from the Acturus Picopure kit (Molecular Devices). The RNA was then isolated from the macrocaps as per kit instructions (Picopure kit, Arcturus). In brief, total cellular extract and ethanol were added to a preconditioned purification column. After incubation, the sample was centrifuged and the RNA washed twice before elution in $15 \mu \mathrm{L}$ of RNaseand DNase-free water. Total RNA quantity was determined using a Nanodrop spectrophotometer (ND-1000, NanoDrop Technologies Inc., Wilmington, DE; $94 \pm$ $34 \mathrm{ng}$ of total RNA per sample). The RNA quality was determined using a Picochip (Bioanalyzer Agilent Inc., Palo Alto, CA) and the average RNA integrity number from samples was 5 .

The RNA profiling of the samples was conducted at the University of Kentucky Microarray Facility using a bovine Affymetrix GeneChip array (Bovine Gene 1.0 ST Array, Affymetrix, Santa Clara, CA). The preparation of cDNA was conducted using Ovation Pico WTA V2 kit (part no. 3302; NuGEN, San Carlos, CA), and cDNA was labeled using Encore Biotin Module kit (part no. 4200; NuGEN). Labeled products were hybridized to arrays according to the NuGEN Technologies protocol. In summary, the microarrays were hybridized in an Affymetrix 640 hybridization oven at $45^{\circ} \mathrm{C}$ for $16 \mathrm{~h}$ with a $60 \mathrm{rpm}$ rotation. The arrays were washed using stringent and nonstringent wash buffers, stained with streptavidin phycoerythrin and antibody solutions, and scanned using an Affymetrix Genearray scanner.

Data preprocessing was conducted using the robust multi-array average method, and detection of differentially expressed genes (DEG) was determined using ANOVA using Partek Software, version 6.3 (Partek Inc., St. Louis, MO). The model included the fixed effect of tissue, and a Benjamini-Hochberg false discovery rate of 0.1 was applied. In total, 382 genes were differentially expressed between tissues $(P<0.001)$. From this list of DEG, 233 had higher expression values in connective tissue and 149 had higher expression values in epithelial tissue.

The list of DEG was subjected to analysis by the Ingenuity Pathway Analysis (IPA) software (Ingenuity Systems, Mountain View, CA) to identify the

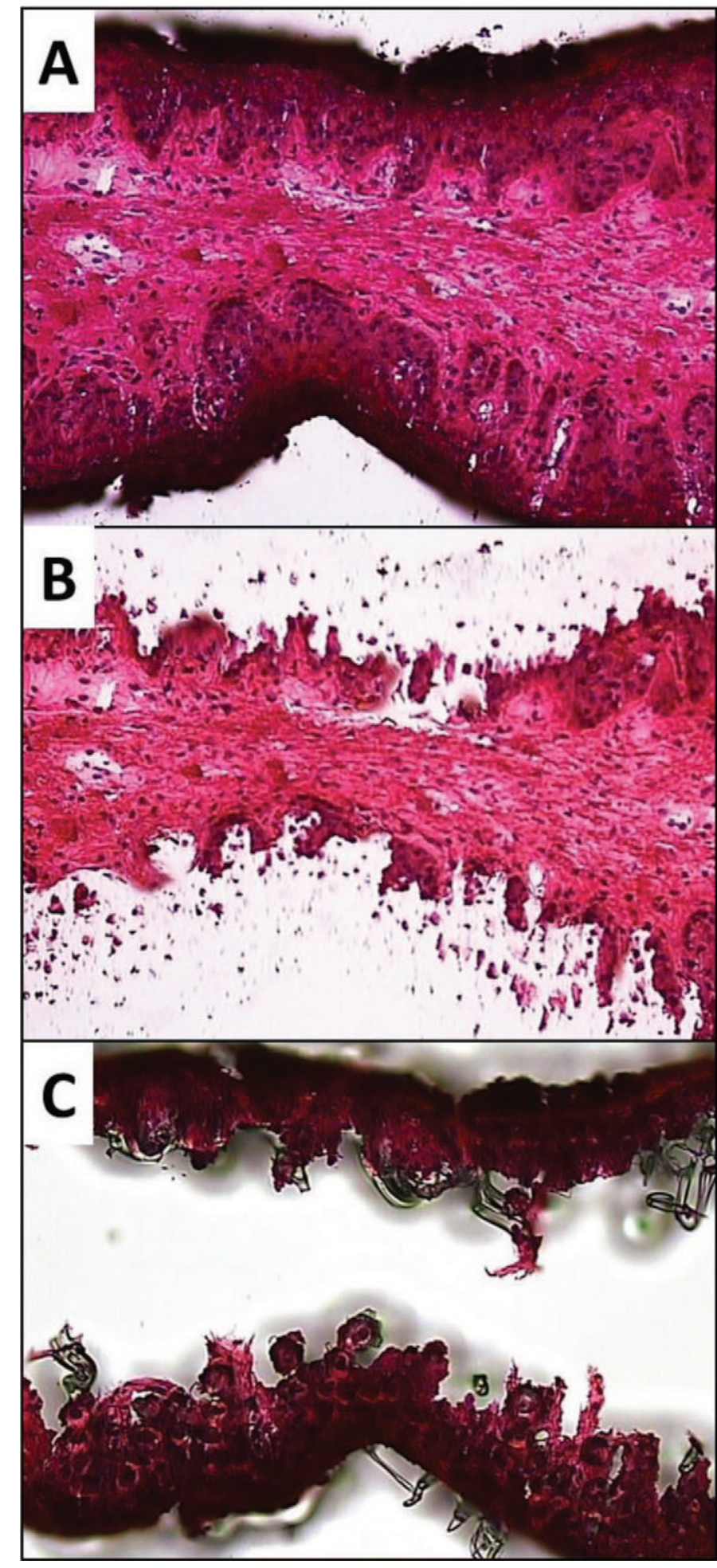

Figure 1. Light micrographs of rumen papillae sections before and after the epithelium was captured using laser capture microdissection (LCM; 200× magnification). Slide of rumen papillae cross-sections (A) before LCM and (B) after LCM; (C) cap with populations of rumen epithelial cells isolated from the slide. Color version available in the online PDF. 
Table 1. List of key differentially expressed genes between connective and epithelial tissues of ruminal papillae, using RNA extracted from tissues types collected by laser capture microdissection

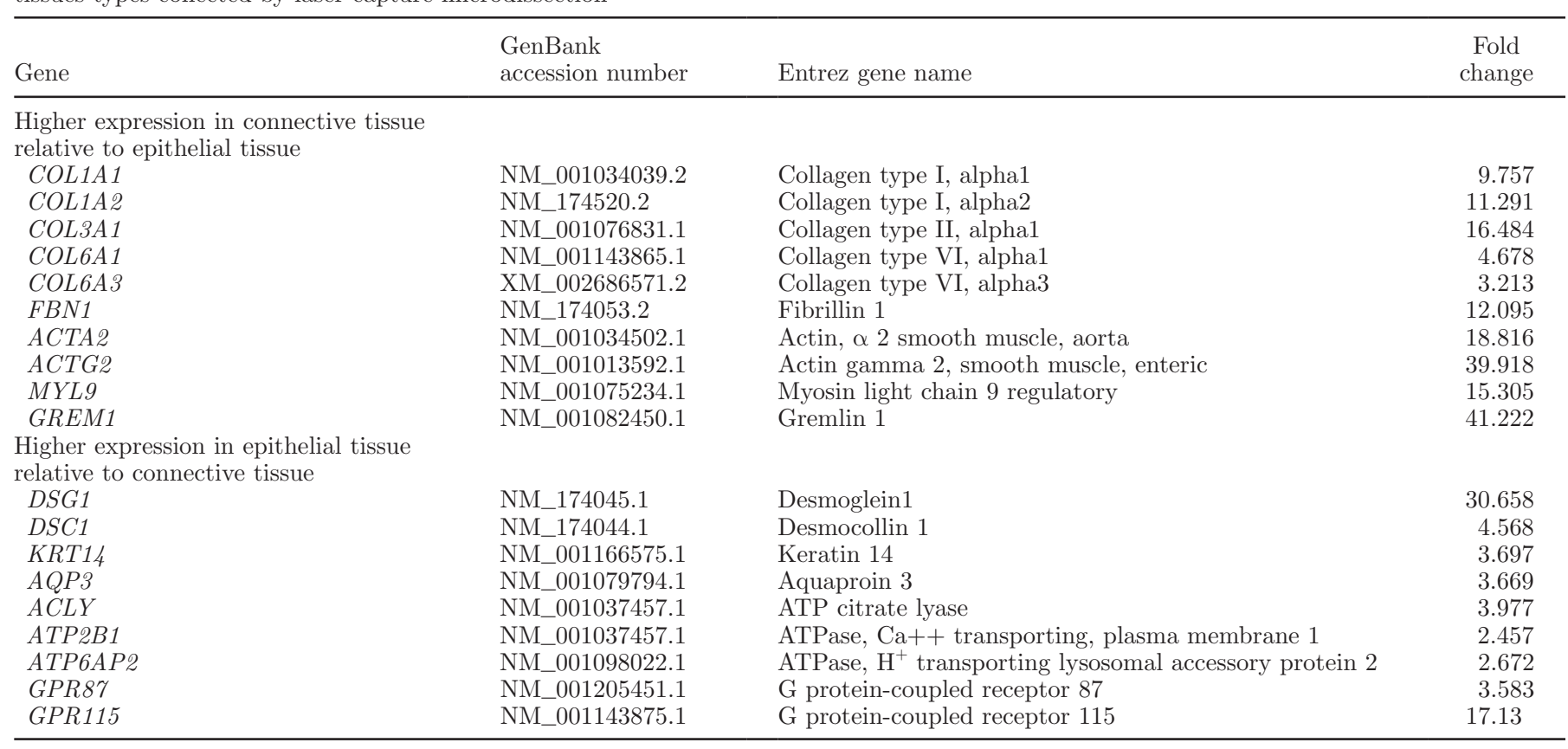

biological functions, canonical pathways, and networks enriched with genes differentially expressed between rumen epithelial and connective transcriptomes. Then, the Affymetrix knowledge base was selected and gene functions were determined using National Center for Biotechnology Information Entrez Gene (http://www. ncbi.nlm.nih.gov/gene). The IPA program works by overlaying the data set with global molecular networks created by an IPA knowledge base. To determine if an association exists between the genes in the data set and a specific canonical pathway, IPA uses a Fisher's exact test to calculate the significance. For uncovering significant gene networks, IPA assigns a score to each biological network, which is calculated by determining the ratio between the number of focus genes in the network and the total genes in the network. The probability of finding this ratio in a given network by chance is determined using a Fisher's exact test, and $P$-values were corrected for multiple testing.

A summary of DEG between the rumen epithelium and connective tissue in biopsied rumen papillae is given in Table 1. Overall, the fold changes of the expression values between tissue types were large (up to 40-fold). Connective tissue was enriched with genes involved in cell structure and extracellular matrix composition such as collagen, actin, and myosin. In contrast, the epithelium was enriched in genes involved in epithelialspecific functions such as tight junction genes, aquaporins, and metabolism. The largest relative expression in rumen epithelial tissue was desmoglein 1 (31-fold), which has been previously determined to be differentially expressed during grain-induced acidosis (Steele et al., 2011a).

Ingenuity Pathway Analysis generated a total of 24 significant $(P<0.01)$ canonical pathways, including epithelial adherens junction signaling, calcium signaling, gap junction signaling, acetyl-CoA biosynthesis, tight junction signaling, and remodeling of epithelial adherens junctions. Top networks within the IPA knowledge base are primarily built on experiments from nonruminant tissues and cells due to the lack of information in ruminants. Nevertheless, there were 20 significant networks and the top 5 had IPA scores above 34 and more than 22 focus molecules. The top functions $(P<0.01)$ within the networks included connective tissue disorders, dermatological diseases and conditions, gastrointestinal disease, tissue morphology, and tissue development. These results suggest that microdissection of epithelial and connective fractions from whole papillae generated unique cell populations characterized by distinct gene expression signatures.

Laser capture microdissection before functional genomic profiling has been utilized in medical (Curran et al., 2000) and plant-based research (Nakazono et al., 2003). Conversely, very few studies have used LCM before functional genomic profiling in animal sciences. Other methodologies have been developed to overcome the heterogeneity challenge such as xenografts and flow cytometry but these require extensive disruption of the original structure of tissue. With respect to the rumen 
tissue research, cell culture techniques control cellular heterogeneity; however, the interpretation of these in vitro results is challenging considering the organizational structure of the epithelium. Another approach recently used to study the expression of genes in rumen tissue at the protein level is semiquantitative immunofluorescence microscopy (Penner et al., 2011). The major disadvantage of semiquantitative immunofluorescence microscopy is the low throughput as proteins must be measured individually.

The development of an LCM protocol is a step forward in the process of overcoming the issues with heterogeneity of rumen tissue samples. To date, only one study in dairy cows has used LCM to study specific stromal and epithelial cells in mammary tissue (Casey et al., 2011). In this study, the epithelial cells were enriched in genes associated with metabolism and secretion, and the stromal cells were enriched in genes associated with extracellular matrix composition and cellular remodeling. These pathways are similar to the findings of this study and highlight the major functional and transcriptomic differences in whole tissue that should be considered when evaluating gene expression data. In addition to improving confidence in the starting material, the tissue collected by LCM can be analyzed using a variety of profiling techniques such as transcriptomics, proteomics, and metabolomics.

Despite the clear advantages of using LCM, some challenges need to be overcome when using this technique in rumen physiology. First, the rumen, like the epithelium, is heterogeneous in nature and LCM uses fewer cells than conventional approaches. Therefore, the importance of sampling multiple papillae per site should not be overlooked. An aspect of LCM that is not discussed in the literature is relevance of the total number of cells and thus RNA collected from the tissue section. Most studies require the pooling of several caps to collect a minimum of $20 \mathrm{ng}$ of RNA for microarray analysis (Casey et al., 2011). In the case of rumen tissue, up to 500 cells from the epithelial and connective tissue fractions were collected per cap, thereby yielding $94 \pm 34 \mathrm{ng}$ of RNA per sample. However, isolating specific cell types within the epithelium, such as basale, spinosum, and granulosum cells, may provide a challenge in collecting enough RNA to conduct transcriptomic profiling.

In summary, the LCM technique improves our ability to examine the molecular profile of a defined target cell population identified through histology. The results from this study show that it is possible to dissect specific cell types from rumen tissue and highlight the importance of examining the roles of cell types when studying mechanisms and designing therapies that promote rumen epithelial function.

\section{ACKNOWLEDGMENTS}

We thank the Agriculture and Agri-Food Canada-Internationalization of Research and Development program (Ottawa, ON, Canada) and the Natural Sciences and Engineering Research Council of Canada (Ottawa, ON, Canada) for funding this project. As well, special thanks go to Caroline O'Neil (University of Western Ontario) and Julie Kim (University of Guelph) for their helpful advice.

\section{REFERENCES}

Baldwin, R. L. 1998. Use of isolated ruminal epithelial cells in the study of rumen metabolism. J. Nutr. 128:293S-296S.

Canadian Council on Animal Care. 1993. Guide to the Care and Use of Experimental Animals. Canadian Council on Animal Care, Ottawa, ON, Canada.

Casey, T., H. Dover, J. Liesman, L. DeVries, M. Kiupel, M. VandeHaar, and K. Plaut. 2011. Transcriptome analysis of epithelial and stromal contributions to mammogenesis in three week prepartum cows. PLoS ONE 6:e22541.

Connor, E. E., R. L. Baldwin VI, C. J. Li, R. W. Li, and H. Chung. 2013. Gene expression in bovine rumen epithelium during weaning identifies molecular regulators of rumen development and growth. Funct. Integr. Genomics 13:133-142.

Curran, S., J. A. McKay, H. L. McLeod, and G. I. Murray. 2000. Laser capture microscopy. Mol. Pathol. 53:64-68.

Dionissopoulos, L., A. Laarman, O. AlZahal, S. Greenwood, M. A. Steele, J. C. Plaizier, J. C. Matthews, and B. W. McBride. 2013. Butyrate-mediated genomic changes involved in non-specific host defenses, matrix remodeling and the immune response in the rumen epithelium of cows afflicted with subacute ruminal acidosis. Am. J. Anim. Vet. Sci. 8:8-27.

Gäbel, G., J. R. Aschenbach, and F. Muller. 2002. Transfer of energy substrates across the ruminal epithelium: Implications and limitations. Anim. Health Res. Rev. 3:15-30.

Loor, J. J., M. Bionaz, and J. K. Drackley. 2013. Systems physiology in dairy cattle: Nutritional genomics and beyond. Annu. Rev. Anim. Sci. 1:365-392.

Nakazono, M., F. Qiu, L. A. Borsuk, and P. S. Schnable. 2003. Laser-capture microdissection, a tool for the global analysis of gene expression in specific plant cell types: Identification of genes expressed differentially in epidermal cells or vascular tissues of maize. Plant Cell 15:583-596.

Penner, G. B., M. A. Steele, J. R. Aschenbach, and B. W. McBride. 2011. Ruminant Nutrition Symposium: Molecular adaptation of ruminal epithelia to highly fermentable diets. J. Anim. Sci. 89:1108-1119.

Steele, M. A., J. Croom, M. Kahler, O. AlZahal, S. E. Hook, K. Plaizier, and B. W. McBride. 2011a. Bovine rumen epithelium undergoes rapid structural adaptations during grain-induced subacute ruminal acidosis. Am. J. Physiol. Regul. Integr. Comp. Physiol. 300:R1515-R1523.

Steele, M. A., G. Vandervoort, O. AlZahal, S. E. Hook, J. C. Matthews, and B. W. McBride. 2011b. Rumen epithelial adaptation to high-grain diets involves the coordinated regulation of genes involved in cholesterol homeostasis. Physiol. Genomics 43:308-316. 